\title{
Editorial
}

\section{Mathematical Tools of Soft Computing 2014}

\author{
Ker-Wei Yu, ${ }^{1}$ Yang Xu, ${ }^{2}$ and Jer-Guang Hsieh ${ }^{3}$ \\ ${ }^{1}$ Department of Marine Engineering, National Kaohsiung Marine University, Kaohsiung 81157, Taiwan \\ ${ }^{2}$ Department of Applied Mathematics, Southwest Jiaotong University, Chengdu, Sichuan 610031, China \\ ${ }^{3}$ Department of Electrical Engineering, I-Shou University, Kaohsiung 84001, Taiwan
}

Correspondence should be addressed to Ker-Wei Yu; kwyu@webmail.nkmu.edu.tw

Received 27 October 2014; Accepted 27 October 2014

Copyright (C) 2015 Ker-Wei Yu et al. This is an open access article distributed under the Creative Commons Attribution License, which permits unrestricted use, distribution, and reproduction in any medium, provided the original work is properly cited.

It is an important and promising topic that applies the soft computing techniques on engineering problems. Many successful applications in diverse fields using the soft computing techniques have been reported. This special issue represents the recent developments of the mathematical tools and algorithms of soft computing to solve formal and practical problems arising from engineering areas. We have collected thirty papers in this special issue, which are summarized as follows.

In the paper " $A$ hybrid metaheuristic-based approach for the aerodynamic optimization of small hybrid wind turbine rotors" by J. F. Herbert-Acero et al., the authors present a novel framework for the aerodynamic design and optimization of blades for small horizontal axis wind turbines. Based on the experimental results, improvements in terms of the aerodynamic efficiency, the cut-in wind speed, and the amount of material used during the manufacturing process can be achieved.

The opposition-based learning strategy and free search operator are embedded into the krill herd optimization algorithm to form a new opposition-based free search krill herd optimization algorithm for function learning problems in the paper "A free search krill herd algorithm for functions optimization" by L. Li et al. Fourteen benchmark functions were tested. It is claimed that, compared with some other evolutionary computation algorithms, the proposed algorithm shows better performance and robustness.

In the paper "A hybrid optimized weighted minimum spanning tree for the shortest intrapath selection in wireless sensor network" by M. Saravanan and M. Madheswaran, a novel hybrid evolutionary algorithm, called bee algorithmsimulated annealing weighted minimal spanning tree routing, is proposed in which randomly deployed sensor nodes are split into the best possible number of independent clusters with cluster head and optimal route. Some numerical simulations are given to illustrate the proposed method.

A novel adaptive elite-based PSO approach is proposed in "A novel adaptive elite-based particle swarm optimization applied to VAR optimization in electric power systems" by Y.Y. Hong et al. A realistic loss minimization problem in an electric power system is studied to show the robustness of the proposed method.

A new method for rigid video object recognition is presented in the paper "An innovative SIFT-based method for rigid video object recognition" by J. Yu et al. It is claimed that the new method can improve greatly the completeness of the feature database of the target video object automatically, which in turn increases drastically the ratio of correct recognition, at the expense of more affordable computation time in millisecond level.

In the paper "Attribute extended algorithm of latticevalued concept lattice based on congener formal context" by L. Yang and Y. Xu, the definitions of attribute extended formal context and congener formal context are given. Under some mild condition, necessary and sufficient conditions of forming attribute values are derived. Based on these conditions, the algorithms of generating lattice-valued congener formal context and establishing concept lattice are developed, by which one can construct algorithms for lattice-valued concept lattices in distributed and parallel systems. 
The paper entitled "Comparing the selected transfer functions and local optimization methods for neural network flood runoff forecast" by P. Maca et al. analyzes the influence of the selection of the transfer functions and the training algorithms on neural network for flood runoff forecast. Nine of the most significant flood events, caused by the extreme rainfall, were selected from 10 years of measurements on small headwater catchment in the Czech Republic, and flood runoff forecast was investigated using the extensive set of multilayer perceptrons with one hidden layer of neurons.

A two-dimensional continuous genetic algorithm is proposed to numerically solve the Stokes and nonlinear Navier Stokes problems in "Continuous genetic algorithm as a novel solver for Stokes and nonlinear Navier Stokes problems" by Z. S. Abo-Hammour et al. The simulation results showed that the proposed continuous genetic algorithm has an excellent accuracy as compared to some other numerical solvers.

The paper "Dealing with nonregular shapes packing” by B. A. Júnior et al. addresses the irregular strip packing problem, a particular two-dimensional cutting and packing problem in which convex and nonconvex shapes (polygons) have to be packed onto a single rectangular object. An approach is proposed that prescribes the integration of a metaheuristic engine (i.e., genetic algorithm) and a placement rule (i.e., greedy bottom-left rule). Computational experiments performed on standard benchmark problems, as well as practical case studies developed in the ambit of a large textile industry, are reported and discussed.

A new derivative-based trapezoid rule for the RiemannStieltjes integral is proposed in the paper "Derivative-based trapezoid rule for the Riemann-Stieltjes integral" by W. Zhao and Z. Zhang. This new rule may increase two orders of precision over the trapezoid rule for the Riemann-Stieltjes integral. The rationality of the generalization of derivativebased trapezoid rule for Riemann-Stieltjes integral is also demonstrated.

Network-based control systems have become emerging technologies in the control of nonlinear systems over the past few years. The paper entitled "Discrete globalised dual heuristic dynamic programming in control of the two-wheeled mobile robot" by M. Szuster and Z. Hendzel focuses on the implementation of the approximate dynamic programming algorithm in the network-based tracking control system of the two-wheeled mobile robot, Pioneer 2-DX. The proposed discrete tracking control system consists of the globalised dual heuristic dynamic programming algorithm, the PD controller, the supervisory term, and an additional control signal. The performance of the proposed control system was verified by a series of computer simulations and experiments realised using the wheeled mobile robot Pioneer 2-DX.

Energy consumption has become a more and more important issue in computer systems. In the paper "Energyaware real-time task scheduling for heterogeneous multiprocessors with particle swarm optimization algorithm" by W. Zhang et al., the authors first formulate and describe the energy-aware real-time task scheduling problem in heterogeneous multiprocessors. Then they propose a particle swarm optimization based algorithm, which can successfully reduce the energy cost and the time for searching for feasible solutions. Some experimental results are provided.

In the paper "Expert system for competences evaluation $360^{\circ}$ feedback using fuzzy logic" by A. A. A. Lasserre et al., the authors propose an expert performance evaluation system based on a fuzzy logic model, with competences $360^{\circ}$ feedback oriented to human behavior. The model was validated in the administrative department of a real Mexican manufacturing company. The experimental results showed that the proposed method has better performance over some other traditional $360^{\circ}$ performance evaluation methods.

In the paper "Extracting credible dependencies for averaged one-dependence estimator analysis" by L. Wang et al., the authors present a new type of semi-Naive Bayesian operation, which selects superparent attributes by building maximum weighted spanning tree and removes highly correlated children attributes by functional dependency and canonical cover analysis. Some experimental comparisons are made on UCI datasets.

Surrogate models can be used, often in conjunction with evolutionary algorithms, in optimization when it is expensive to test candidate solutions. In the paper "Geometric generalisation of surrogate model-based optimisation to combinatorial and program spaces" by Y.-H. Kim et al., it is shown how radial basis functions can provide a generalised surrogate model for combinatorial problems which have a geometric solution representation, through the conversion of that representation to a different metric space.

In the paper "Hybrid soft computing schemes for the prediction of import demand of crude oil in Taiwan" by Y. E. Shao et al., single-stage and two-stage hybrid forecasting models are proposed for the prediction of imported crude oil in Taiwan. Real data set of crude oil in Taiwan over the period of 1993-2010 was tested.

In recent years, grammatical evolution has been used as a representation of genetic programming, which has successfully been applied in many optimization problems. In the paper "Improving the bin packing heuristic through grammatical evolution based on swarm intelligence" by M. A. Sotelo-Figueroa et al., the authors propose using particle evolutionary swarm optimization (PESO) and particle swarm optimization (PSO) in the frame of grammatical evolution as strategies to generate heuristics that solve the bin packing problems.

The supply chain is a major issue in a global market. In the paper "Methodology for supply chain integration: a case study in the artisan industry of footwear" by J. R. PérezGallardo et al., a methodology is proposed for integrating the supply chain that can be applied in any type of business. To demonstrate its relevance, a case study is performed at a handmade shoe company.

Due to the fact that the bit reversal process requires considerable processing time of the fast Fourier transformation (FFT) algorithm, it is vital to optimize the bit reversal algorithm (BRA). An efficient BRA with multiple memory structures is proposed in the paper "Multiple memory structure bit reversal algorithm based on recursive patterns of bit reversal permutation" by K. K. L. B. Adikaram et al. Some numerical examples are given to illustrate the proposed method. 
The decision support systems are usually created to help decision makers to make complex decisions. In the paper "OrclassWeb: a tool based on the classification methodology ORCLASS from verbal decision analysis framework" by T. C. S. Machado et al., a new tool, called OrclassWeb, is developed to reproduce the procedure to apply the verbal decision analysis methodology ORCLASS.

A hesitant fuzzy linguistic term set (HFLTS) is very useful to express people's hesitancy in practical decision making problems. In the paper "Pairwise comparison and distance measure of hesitant fuzzy linguistic term sets" by H.-C. Huang and X. Yang, the authors present a comparison method for HFLTSs based on pairwise comparisons of each linguistic term in the two HFLTSs. Then, a distance measure method based on the pairwise comparison matrix of HFLTSs is proposed, and this distance is proved to be equal to the distance of the average values of HFLTSs, which makes the distance measure much more simple. Finally, the pairwise comparison and distance measure methods are utilized to develop two multicriteria decision making approaches under hesitant fuzzy linguistic environments.

Tourist distribution, a vector reflecting the tourist number of scenic spots in a certain period of time, serves as the foundation for a scenic spot manager to make a schedule scheme. In the paper "Real-time forecast of tourists distribution based on the improved k-means method" by P. Ren et al., a forecast model based on improved $k$-means method is provided to forecast tourist distribution. A case study of Jiuzhai Valley is adopted to illustrate the effectiveness of the proposed forecast model.

The trajectory optimization problem subject to terminal impact time and angle specifications is investigated in the paper "Receding horizon trajectory optimization with terminal impact specifications" by L. Zhang et al. A receding horizon optimization strategy is implemented to reject the errors caused by the motion of a surface target. Several simulations were performed to validate the proposed method.

In the paper "The lattice-valued Turing machines and the lattice-valued type 0 grammars" by J. Tang et al., the authors introduce a new concept of the $I$-valued Turing machine. It can be used to characterize recognition, natural language processing, and dynamic characteristics.

The global warming will lead to rising temperature in Tibetan plateau, and it has severe effects to the long-term stability of frozen soil roadbed. In the paper "Simulation analysis of the long-term stability for frozen soil roadbed" by $\mathrm{X}$. Liu et al., a numerical simulation model is established for the frozen soil roadbed in Tibetan plateau.

The Triple I method for the model of intuitionistic fuzzy modus tollens (IFMT) satisfies the property of local reductivity but not the reductivity. In order to improve the quality of the Triple I method for the lack of reductivity, a new approximate reasoning method is proposed for IFMT problem in the paper "The dual Triple I methods of FMT and IFMT" by L. Yan and Z. Mucong.

In the paper "A MOLP method for solving fully fuzzy linear programming with LR fuzzy parameters" by X.-P. Yang et al., a new class of fully fuzzy linear programming (FFLP) problems with LR fuzzy parameters are studied. The FFLP is converted into a multiobjective linear programming (MOLP) according to the order relation for comparing the LR at fuzzy numbers. Moreover, the classical fuzzy programming method is modified and used to solve the MOLP problems. A numerical example is given to illustrate the feasibility of the proposed method.

In the paper "A multiobjective optimization algorithm based on discrete bacterial colony chemotaxis" by Z. Lu et al., a discrete bacterial colony chemotaxis algorithm is proposed to solve multiobjective optimization problems. Some numerical results are given to demonstrate the effectiveness of the proposed method.

A prime objective in constructing data streaming mining models is to achieve good accuracy, fast learning, robustness to noise, and a compact tree size. In the paper "A lightweight data preprocessing strategy with fast contradiction analysis for incremental classifier learning" by S. Fong et al., a new data preprocessing strategy is proposed which is suitable for the progressive purging of noisy data from the training dataset without the need to process the whole dataset at one time. This strategy is tested via a computer simulation to show the significant benefit of allowing for the dynamic removal of bad records in the incremental classifier learning process.

In the paper "Multisegment scheme applications to modified Chebyshev Picard Iteration method for highly elliptical orbits" by D. Kim et al., a modified Cheyshev Picard iteration method is proposed for solving orbit propagation initial/ boundary value problems. Cosine sampling techniques are used to reduce Runge's phenomenon that plagues many series approximations. The authors suggest a multisegment method to obtain accurate solutions overall regardless of initial positions and eccentricity by dividing the given orbit into two or more segments.

\section{Acknowledgments}

We wish to express our sincere appreciation to the authors for their excellent contributions. The hard work of all reviewers is greatly acknowledged. Without them, this special issue can never become possible.

Ker-Wei Yu Yang Xu

Jer-Guang Hsieh 


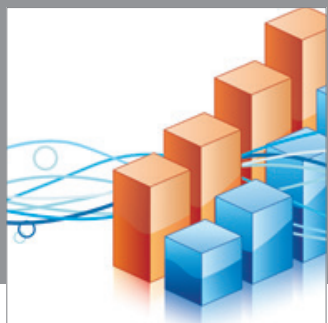

Advances in

Operations Research

mansans

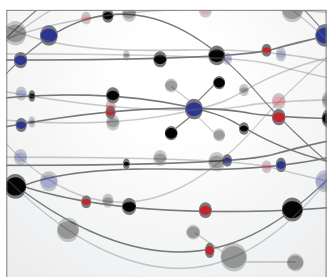

The Scientific World Journal
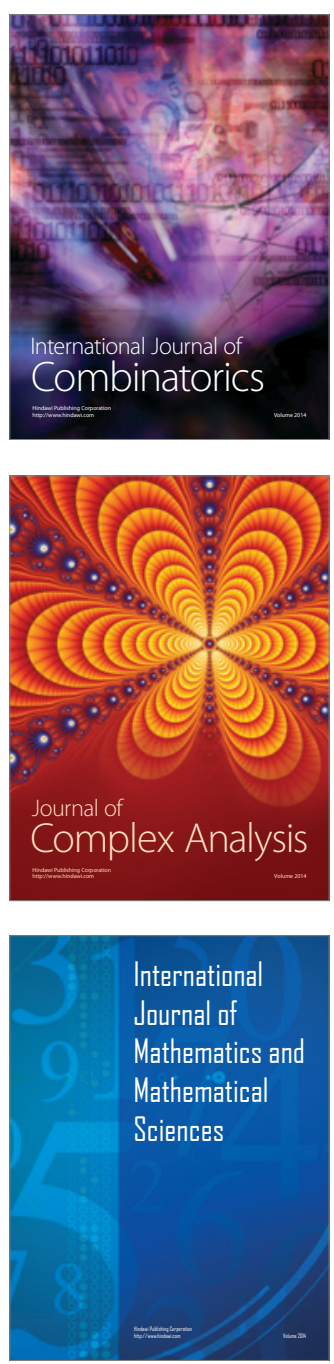
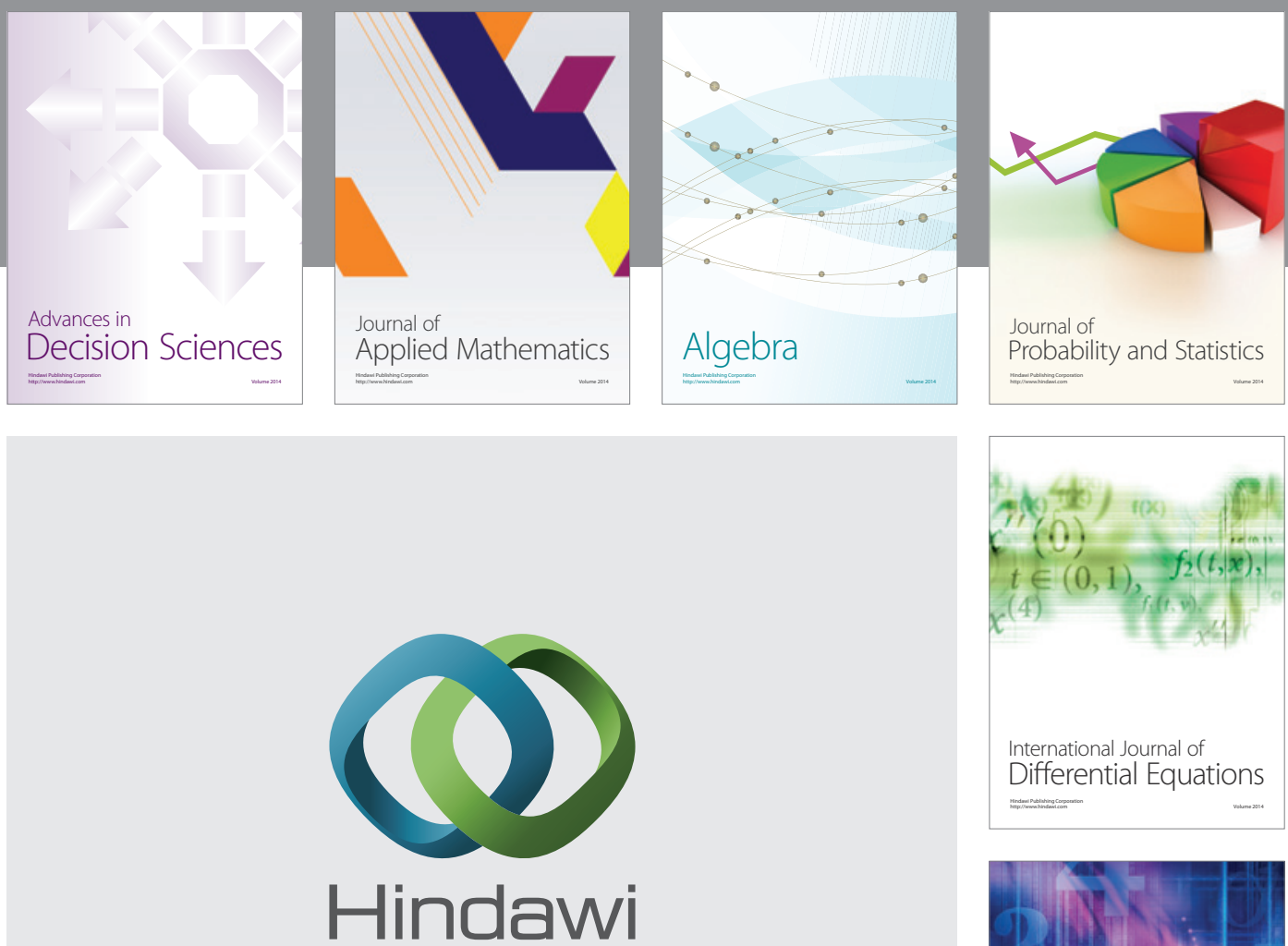

Submit your manuscripts at http://www.hindawi.com
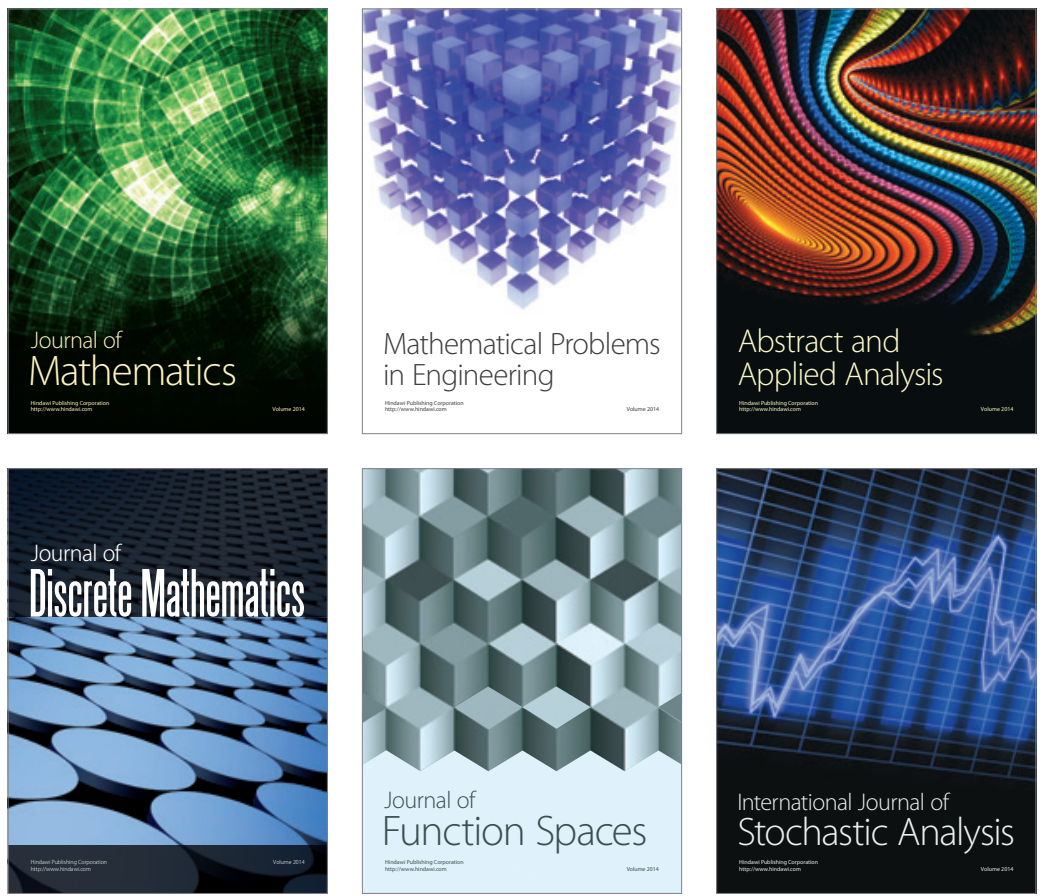

Journal of

Function Spaces

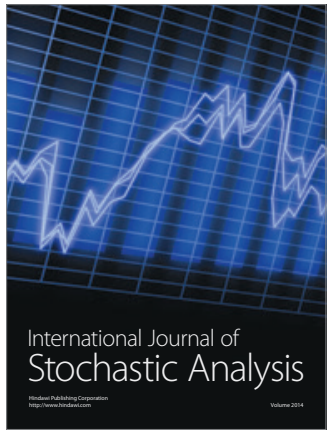

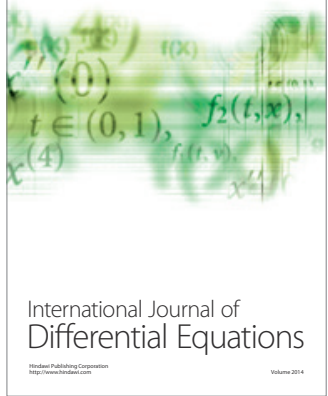
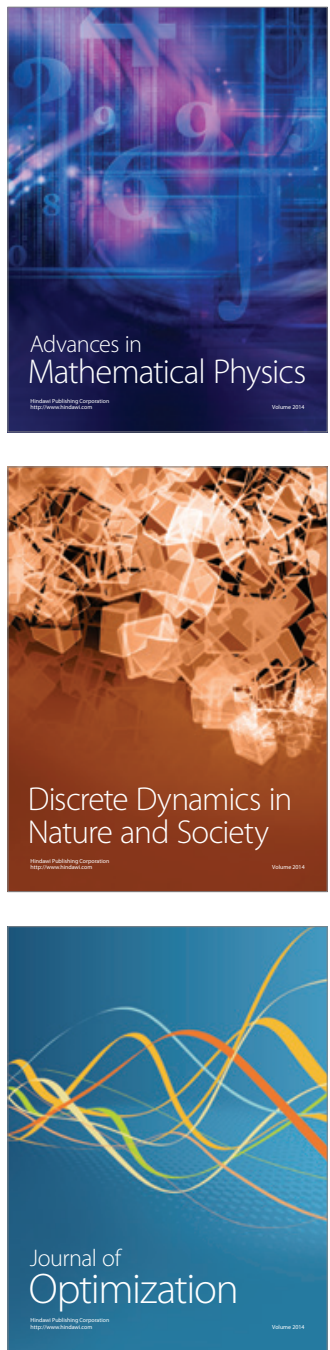